\title{
On the Weisfeiler-Leman Dimension of Fractional Packing
}

\author{
V. Arvind, Frank Fuhlbrück, Johannes Köbler ${ }^{\dagger}$, Oleg Verbitsky ${ }^{\dagger \dagger}$
}

\begin{abstract}
The $k$-dimensional Weisfeiler-Leman procedure $(k$-WL), which colors $k$ tuples of vertices in rounds based on the neighborhood structure in the graph, has proven to be immensely fruitful in the algorithmic study of Graph Isomorphism. More generally, it is of fundamental importance in understanding and exploiting symmetries in graphs in various settings. Two graphs are $k$-WLequivalent if the $k$-dimensional Weisfeiler-Leman procedure produces the same final coloring on both graphs. 1-WL-equivalence is known as fractional isomorphism of graphs, and the $k$-WL-equivalence relation becomes finer as $k$ increases.

We investigate to what extent standard graph parameters are preserved by $k$-WL-equivalence, focusing on fractional graph packing numbers. The integral packing numbers are typically NP-hard to compute, and we discuss applicability of $k$-WL-invariance for estimating the integrality gap of the LP relaxation provided by their fractional counterparts.
\end{abstract}

\section{Introduction}

The 1-dimensional version of the Weisfeiler-Leman procedure is the classical color refinement applied to an input graph $G$. Each vertex of $G$ is initially colored by its degree. The procedure refines the color of each vertex $x \in V(G)$ in rounds, using the multiset of vertex colors in the neighborhood of $x$. In the 2-dimensional version [43], all vertex pairs $(x, y) \in V(G) \times V(G)$ are classified by a similar procedure of coloring them in rounds. The extension of this procedure to a classification of all $k$-tuples of $G$ is due to Babai (see historical overview in [4, 9]) and is known as the $k$-dimensional Weisfeiler-Leman procedure, abbreviated as $k$-WL. Graphs $G$ and $H$ are said to be $k$-WL-equivalent (denoted $G \equiv_{k \text {-WL }} H$ ) if they are indistinguishable by $k$-WL.

\footnotetext{
${ }^{*}$ The Institute of Mathematical Sciences (HBNI), Chennai, India.

${ }^{\dagger}$ Institut für Informatik, Humboldt-Universität zu Berlin, Germany.

${ }_{\ddagger}^{\ddagger}$ Supported by DFG grant KO 1053/8-1. On leave from the IAPMM, Lviv, Ukraine.
} 
The WL invariance of graph parameters. Let $\mathcal{G}$ denote the set of all graphs. A graph parameter is a function $\pi$ defined on $\mathcal{G}$ such that $\pi(G)=\pi(H)$ whenever $G$ and $H$ are isomorphic.

We say that $\pi$ is $k$-WL-invariant if the equality $\pi(G)=\pi(H)$ is implied even by the weaker condition $G \equiv_{k \text {-WL }} H$.

Definition 1.1. The Weisfeiler-Leman (WL) dimension of a graph parameter $\pi$ is the least positive integer $k$, if it exists, such that for any pairs of graphs $G$ and $H$ that are $k$-WL-indistinguishable we have $\pi(G)=\pi(H)$. If no such $k$ exists, we say that the WL dimension of $\pi$ is unbounded.

Knowing that a parameter $\pi$ has unbounded WL dimension is important from a descriptive complexity perspective, because it implies that $\pi$ cannot be computed by any algorithm expressible in fixed-point logic with counting (FPC), which is a robust framework for study of encoding-invariant (or "choiceless") computations; see the survey [13].

The focus of our paper is on graph parameters with bounded WL dimension. A subset $\mathcal{P} \subseteq \mathcal{G}$ is a graph property if the indicator function $\pi$ of $\mathcal{P}$ is a graph parameter. It is well-known in finite model theory [9] that $\pi$ is $k$-WL-invariant if and only if $\mathcal{P}$ is definable in the infinitary $(k+1)$-variable counting logic $C_{\infty \omega}^{k+1}$. While minimizing the number of variables is a recurring theme in descriptive complexity; see, e.g. [30, 20], our interest in the study of $k$-WL-invariance has an additional motivation: If we know that a graph parameter $\pi$ is $k$-WL-invariant, this gives us information not only about $\pi$ but also about $k$-WL.

Indeed, $k$-WL-invariance admits the following interpretation. We say that a (not necessarily numerical) graph invariant $\pi_{1}$ subsumes a graph invariant $\pi_{2}$ if $\pi_{1}(G) \neq \pi_{1}(H)$ whenever $\pi_{2}(G) \neq \pi_{2}(H)$. That is to say, whenever $\pi_{2}$ distinguishes between graphs $G$ and $H, \pi_{1}$ also does. Let $\mathrm{WL}_{k}(G)$ denote the graph invariant computed by $k$-WL on input $G$. As easily seen, a parameter $\pi$ is $k$-WL-invariant if and only if $\pi$ is subsumed by $\mathrm{WL}_{k}$. Which graph parameters are subsumed by $\mathrm{WL}_{k}$ is of interest even for dimensions $k=1$ and $k=2$, in view of the importance of 1-WL (color refinement) and 2-WL (the original Weisfeiler-Leman algorithm) in isomorphism testing [4, 5] and, more recently, also in other application areas [34, 37. It is known, for example, that the largest eigenvalue of the adjacency matrix has WL dimension 1 (see [41]), and the whole spectrum of a graph has WL dimension 2 (see [14, 24]). Relatedly, Kiefer and Neuen [32] recently proved that $\mathrm{WL}_{2}$ subsumes, in a certain strong sense, the decomposition of a graph into 3-connected components.

Fractional graph parameters. In this paper, we mainly consider fractional graph parameters. Algorithmically, a well-known approach to tackling intractable optimization problems is to consider an appropriate linear programming (LP) relaxation. Many standard integer-valued graph parameters have fractional real-valued analogues, obtained by LP-relaxation of the corresponding 0-1 linear program; see, e.g., the monograph [41]. The fractional counterpart of a graph parameter $\pi$ is 
denoted by $\pi_{f}$. While $\pi$ is often be hard to compute, the fractional parameter $\pi_{f}$ sometimes provides a good polynomial-time computable approximation of $\pi$.

The WL dimension of a natural fractional parameter $\pi_{f}$ is a priori bounded, where natural means that $\pi_{f}$ is determined by an LP which is logically interpretable in terms of an input graph $G$. A striking result of Anderson, Dawar, Holm [1] says that the optimum value of an interpretable LP is expressible in FPC. It follows from the known immersion of FPC into the finite-variable infinitary counting $\operatorname{logic} C_{\infty \omega}^{\omega}=$ $\bigcup_{k=2}^{\infty} C_{\infty \omega}^{k}$ (see [38]), that each such $\pi_{f}$ is $k$-WL-invariant for some $k$. Although this general theorem is applicable to many graph parameters of interest, it is not a priori evident how to extract an explicit value of $k$ from the proof of the theorem, and in any case such a value of $k$ seems unlikely to be optimal.

We are interested in explicit and, possibly, exact bounds for the WL dimension. A first question here would be to pinpoint which fractional parameters $\pi_{f}$ are 1-WL-invariant. This natural question, using the concept of fractional isomorphism [41, can be recast as follows: Which fractional graph parameters are invariant under fractional isomorphisms? It appears that this question has not received adequate attention in the literature. The only earlier result we could find is the 1-WL-invariance of the fractional domination number $\gamma_{f}$ shown in the Ph.D. thesis of Rubalcaba [40].

We show that the fractional matching number $\nu_{f}$ is also a fractional parameter preserved by fractional isomorphism. Indeed, the matching number is an instance of the F-packing number $\pi^{F}$ of a graph, corresponding to $F=K_{2}$. Here and throughout, we use the standard notation $K_{n}$ for the complete graphs, $P_{n}$ for the path graphs, and $C_{n}$ for the cycle graph on $n$ vertices. In general, $\pi^{F}(G)$ is the maximum number of vertex-disjoint subgraphs of $G$ that are isomorphic to the fixed pattern graph $F$. While the matching number is computable in polynomial time, computing $\pi^{F}$ is NP-hard whenever $F$ has a connected component with at least 3 vertices [33], in particular, for $F \in\left\{P_{3}, K_{3}\right\}$. Note that $K_{3}$-packing is the optimization version of the archetypal NP-complete problem Partition Into Triangles [25, GT11]. We show that the fractional $P_{3}$-packing number $\nu_{f}^{P_{3}}$, like $\nu_{f}=\pi_{f}^{K_{2}}$, is 1-WL-invariant, whereas the WL dimension of the fractional triangle packing is 2.

In fact, we present a general treatment of fractional $F$-packing numbers $\pi_{f}^{F}$. We begin in Section 2 with introducing a concept of equivalence between two linear programs $L_{1}$ and $L_{2}$ ensuring that equivalent $L_{1}$ and $L_{2}$ have equal optimum values. Next, in Section 4, we consider the standard optimization versions of Set Packing and Hitting Set [25, SP4 and SP8], two of Karp's 21 NP-complete problems [31. These two generic problems generalize $F$-Packing and Dominating Set respectively. Their fractional versions have thoroughly been studied in hypergraph theory [35, 23]. We observe that the LP relaxations of Set Packing (or Hitting Set) are equivalent whenever the incidence graphs of the input set systems are 1-WL-equivalent. This general fact readily implies Rubalcaba's result [40] on the 1-WL-invariance of the fractional domination number and also shows that, if the pattern graph $F$ has $\ell$ vertices, then the fractional $F$-packing number $\pi_{f}^{F}$ is $k$-WL-invariant for some $k<$ $2 \ell$. This bound for $k$ comes from a logical definition of the instance of Set Packing corresponding to $F$-Packing in terms of an input graph $G$ (see Section 4.3). Though 
the bound is quite decent, it does not need to be optimal. We elaborate on a more precise bound, where we need to use additional combinatorial arguments even in the case of the fractional matching.

We treat the fractional matching separately for expository purposes in Section 4. The general $F$-packing is considered in Section 5, where our main result, Theorem 5.3, includes the aforementioned cases of $F=K_{3}, P_{3}$.

The edge-disjoint version of $F$-Packing is another problem that has intensively been studied in combinatorics and optimization. Since it is known to be NP-hard for any pattern $F$ containing a connected component with at least 3 edges [15], fractional relaxations have received much attention in the literature [16, 27, 44, 45]. We show that our techniques work well also in this case. In particular, the WL dimension of the fractional edge-disjoint triangle packing number $\rho_{f}^{K_{3}}$ is 2 (Theorem 4.6).

Integrality gap via invariance ratio. Furthermore, we discuss the approximate invariance of integral graph parameters expressible by integer linear programs. As a first example, note that the 1-WL-invariance of the fractional matching number $\nu_{f}$ has two consequences. The first follows from the known fact [41, Theorem 2.1.3] that $\nu_{f}(G)=\nu(G)$ if $G$ is bipartite. This equality implies that over bipartite graphs even the integral parameter $\nu$ is 1-WL-invariant; cf. [7, 3].

Another consequence concerns all graphs and is based on Lovász's inequality [23, Theorem 5.21]

$$
\nu_{f}(G) \leq \frac{3}{2}(\tau(G)+\nu(G)) \leq \frac{3}{2} \nu(G)
$$

where $\tau(G)$ is the domination number of a graph $G$. As $\nu_{f}$ is 1 -WL-invariant, it follows that

$$
\nu(G) / \nu(H) \leq 3 / 2
$$

for any pair of nonempty 1-WL-equivalent graphs $G$ and $H$. The bound (2) is tight, as seen for the 1-WL-equivalent graphs $G=C_{6 s}$ and $H=2 s C_{3}$. Consequently, the relationship between $\nu_{f}$ and $\nu$ given by (1) is also tight. This simple example shows that the exact value $k$ of the WL dimension of a fractional parameter $\pi_{f}$, and the discrepancy of the integral parameter $\pi$ over $k$-WL-invariant graphs together yield a lower bound for the precision of approximating $\pi$ by $\pi_{f}$.

Specifically, recall that the maximum

$$
\max _{G} \frac{\pi_{f}(G)}{\pi(G)}
$$

(respectively, $\max _{G} \pi(G) / \pi_{f}(G)$ for minimization problems) is known as the integrality gap of $\pi_{f}$. The integrality gap is important for a computationally hard graph parameter $\pi$, as it bounds how well the polynomial-time computable parameter $\pi_{f}$ approximates $\pi$.

On the other hand, we define the $k$-WL-invariance ratio for the parameter $\pi$ as

$$
\max _{G, H} \frac{\pi(G)}{\pi(H)},
$$


where the quotient is maximized over all $k$-WL-equivalent graph pairs $(G, H)$. If $\pi$ is $k$-WL-invariant, then the $k$-WL-invariance ratio bounds the integrality gap from below. The following question suggests itself: How tight is this lower bound? In this regard, let us look at the fractional domination number $\gamma_{f}$ again.

A general bound by Lovász [35] on the integrality gap of the fractional covering number for hypergraphs implies for a graph $G$ that $\gamma(G) \leq(1+\ln (1+\Delta(G))) \gamma_{f}(G)$, where $\Delta(G)$ denotes the maximum vertex degree of $G$. It follows that the integrality gap for the domination number is at most logarithmic. More precisely,

$$
\frac{\gamma(G)}{\gamma_{f}(G)} \leq 1+\ln n
$$

for a non-empty graph $G$ with $n$ vertices. This results in an LP-based algorithm for approximation of $\gamma(G)$ within a logarithmic factor, which is essentially optimal as $\gamma(G)$ is inapproximable within a factor of $(1-\epsilon) \ln n$ unless NP $\subseteq \operatorname{DTIME}\left(n^{O(\log \log n)}\right)$; see [12]. Recall that $\gamma_{f}$ is 1-WL-invariant. Along with (3), this implies that the 1-WL-invariance ratio of $\gamma$ is at most logarithmic. On the other hand, Chappell et al. [11] have shown that the bound (3) is tight up to a constant factor. In Section [6] we prove an $\Omega(\log n)$ lower bound even for the 1-WL-invariance ratio of $\gamma$ over $n$-vertex graphs. This implies the integrality gap lower bound [11, reproving it from a different perspective. Moreover, our proof provides a solution to Problem 3.4 in [11] asking for an explicit construction of graphs with logarithmic integrality gap for $\gamma_{f}$.

Next, we consider the fractional edge-disjoint triangle packing number $\rho_{f}^{K_{3}}$. A general bound for the integrality gap of the fractional matching number of a hypergraph [22] implies that the integrality gap of $\rho_{f}^{K_{3}}$ is at most 2 (see Theorem 6.1). This yields a polynomial-time algorithm approximating $\rho^{K_{3}}$ within a factor of 2 , which is competitive with the greedy algorithm whose approximation ratio is 3 ; see [45].1. We observe that the upper bound of 2 is sharp, as 2 is also a lower bound for the 2-WL-invariance ratio of $\rho^{K_{3}}$.

Upper bounds for the additive integrality gap of $\rho_{f}^{K_{3}}$ prove to be of considerable interest, implying a PTAS for $\rho^{K_{3}}$ on dense graphs [27, 44]. Motivated by this fact, in Section [6] we obtain a lower bound also for the 2-WL-invariance difference of $\rho^{K_{3}}$.

Related work. Atserias and Dawar 2 have shown that the 1-WL-invariance ratio for the vertex cover number $\tau$ is at most 2. Alternatively, this bound also follows from the 1-WL-invariance of $\nu_{f}$ (which implies the 1-WL-invariance of $\tau_{f}$ as $\tau_{f}=\nu_{f}$ by LP duality) combined with a standard rounding argument. The argument presented in [2] is different2 and alone does not yield 1-WL-invariance of the fractional vertex cover $\tau_{f}$.

\footnotetext{
${ }^{1}$ Though there are approaches [29] giving a better approximation ratio of $\frac{3}{2}+\epsilon$, it is known [19] that there is no polynomial-time approximation scheme (PTAS) for $\rho^{K_{3}}$ unless NP $=\mathrm{P}$.

${ }^{2}$ The approach of [2] is based on constructing weighted graphs $X_{G}$ and $X_{H}$ that are isomorphic if $G \equiv_{1 \text {-WL }} H$. The vertex cover number $\tau(G)$ is estimated from below and from above in terms of weighted vertex covers of $X_{G}$, and $\tau_{f}(G)$ appears as a technical tool in the argument.
} 
The bound of 2 for the 1-WL-invariance ratio of $\tau$ is optimal. Atserias and Dawar [2] also show that the $k$-WL-invariance ratio for $\tau$ is at least $7 / 6$ for each $k$. This implies an unconditional inapproximability result for Vertex Cover in the model of encoding-invariant computations expressible in FPC. It remains open if similar lower bounds on the invariance ratios can be shown for Dominating Set and Triangle Packing. For each parameter $\pi$ under consideration, we mainly focus on $k$-WL-invariance for $k$ equal to the WL dimension of $\pi$. This focus is motivated by applications to proving lower bounds for the integrality gap between $\pi$ and $\pi_{f}$ as discussed above.

\section{Reductions between linear programs}

A linear program (LP) is an optimization problem of the form "maximize (or minimize) $a^{t} x$ subject to $M x \leq b$ ", where $a \in \mathbb{R}^{n}, b \in \mathbb{R}^{m}$, and $M \in \mathbb{R}^{m \times n}$ is an $m \times n$ matrix. The variable $x$ varies over all vectors in $\mathbb{R}^{n}$ with nonnegative entries (which we denote by $x \geq 0$ ). Any vector $x$ satisfying the constraints $M x \leq b, x \geq 0$ is called a feasible solution and the function $x \mapsto a^{t} x$ is called the objective function. We denote an LP with parameters $a, M, b$ by $L P(a, M, b, o p t)$, where opt $=$ min if the problem is minimization, and opt $=\max$ if it is maximization. The optimum value of the objective function over all feasible solutions is called the value of the program $L=L P(a, M, b, o p t)$ and denoted by $\operatorname{val}(L)$.

Our goal now is to introduce an equivalence relation between LPs ensuring equality of their values. We begin with a motivating discussion.

Isomorphic and isometric LPs. By duality, we can restrict our attention to maximization problems. For the present discussion, we consider linear programs $L_{1}=L P(a, M, b, \max )$ such that all feasible solutions $x$ satisfy $M x=b$. This is ensured by the standard construction of augmenting the LP with slack variables. With this LP we associate the linear transformation $\alpha: \mathbb{R}^{n} \rightarrow \mathbb{R}^{m}$ defined by $\alpha(x)=M x$. Let $L_{2}=L P(c, N, d$, max $)$ be another LP with associated linear transformation $\beta: \mathbb{R}^{n} \rightarrow \mathbb{R}^{m}$, where $\beta(x)=N x$.

We say that $L_{1}$ and $L_{2}$ are isomorphic if the following two conditions hold:

(A) There is a permutation $\phi$ of the $n$ variables $x_{1}, \ldots, x_{n}$ and a permutation $\psi$ of the $m$ equations such that if $Z \in \mathbb{R}^{n \times n}$ and $Y \in \mathbb{R}^{m \times m}$ are the permutation matrices corresponding to $\phi$ and $\psi$ respectively, then the linear transformations $\phi(x)=Z x$ and $\psi(w)=Y w$ make the following diagram

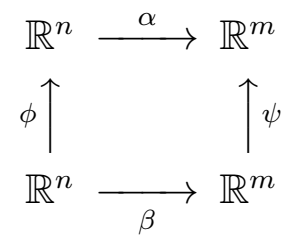

commute. That is, $\alpha \phi=\psi \beta$. 
The second condition is

(B) $\phi(c)=a$ and $\psi(d)=b$.

Suppose that $L_{1}$ and $L_{2}$ are isomorphic. If $x$ is a feasible solution of $L_{2}$, which means $\beta(x)=d$, then $x^{\prime}=\phi(x)$ is a feasible solution of $L_{1}$. Indeed,

$$
\alpha\left(x^{\prime}\right)=\alpha(\phi(x))=\psi(\beta(x))=\psi(d)=b .
$$

Moreover,

$$
a^{t} x^{\prime}=\phi(c)^{t} \phi(x)=c^{t} x,
$$

implying that $\operatorname{val}\left(L_{1}\right) \geq \operatorname{val}\left(L_{2}\right)$. Since the isomorphism of LPs is clearly an equivalence relation, it follows by symmetry that $\operatorname{val}\left(L_{1}\right)=\operatorname{val}\left(L_{2}\right)$.

More generally, suppose there are matrices $Y \in \mathbb{R}^{n \times m}$ and $Z \in \mathbb{R}^{m \times n}$ such that they satisfy the orthogonality conditions $Y^{t} Y=I_{m}$ and $Z^{t} Z=I_{n}$ (where $I_{n}$ is the $n \times n$ identity matrix). Then we say $L_{1}$ and $L_{2}$ isometric if conditions (A) and (B) are fulfilled with $\phi(x)=Z x$ and $\psi(w)=Y w$. Equations (4) and (5) remain true as

$$
\phi(c)^{t} \phi(x)=(Z c)^{t} Z x=c^{t} Z^{t} Z x=c^{t} I_{n} x=c^{t} x .
$$

Hence, $\operatorname{val}\left(L_{1}\right)=\operatorname{val}\left(L_{2}\right)$ also holds for isometric $L_{1}$ and $L_{2}$. This follows from the symmetry of the isometry relation, which in its turn follows from the orthogonality conditions satisfied by matrices $Y$ and $Z$.

We now consider an equivalence concept for LPs, more general than the orthogonality conditions, ensuring the equality of LP values.

Equivalence of LPs. Let $L_{1}=L P(a, M, b, o p t)$ and $L_{2}=L P(c, N, d, o p t)$ be linear programs (in general form), where $a, c \in \mathbb{R}^{n}, b, d \in \mathbb{R}^{m}, M, N \in \mathbb{R}^{m \times n}$ and opt $\in\{\min , \max \}$. We say that $L_{1}$ reduces to $L_{2}\left(L_{1} \leq L_{2}\right.$ for short $)$, if there are matrices $Y \in \mathbb{R}^{m \times m}$ and $Z \in \mathbb{R}^{n \times n}$ such that

- $Y, Z \geq 0$

- $a^{t} Z \diamond c^{t}$, where $\diamond= \begin{cases}\leq, & \text { if } \text { opt }=\min \\ \geq, & \text { if } \text { opt }=\max \end{cases}$

- $M Z \leq Y N$

- $Y d \leq b$

$L_{1}$ and $L_{2}$ are said to be equivalent if $L_{1} \leq L_{2}$ and $L_{2} \leq L_{1}$.

Theorem 2.1. Equivalent linear programs $L_{1}$ and $L_{2}$ have equal values, i.e., val $\left(L_{1}\right)=$ $\operatorname{val}\left(L_{2}\right)$. 
Proof. Let $L_{1}=L P(a, M, b, o p t)$ and $L_{2}=L P(c, N, d$,opt $)$ and assume $L_{1} \leq L_{2}$ via $(Y, Z)$. We show that for any feasible solution $x$ of $L_{2}$ we get a feasible solution $x^{\prime}=Z x$ of $L_{1}$ with $a^{t} x^{\prime} \diamond c^{t} x$, where the relation symbol $\diamond$ is as defined above. Indeed,

$$
M x^{\prime}=\underbrace{M Z}_{\leq Y N} x \leq Y \underbrace{N x}_{\leq d} \leq Y d \leq b \text { and } a^{t} x^{\prime}=\underbrace{a^{t} Z}_{\diamond c^{t}} x \diamond c^{t} x .
$$

Thus, $L_{1} \leq L_{2}$ implies $\operatorname{val}\left(L_{1}\right) \diamond \operatorname{val}\left(L_{2}\right)$ and the theorem follows.

Note that isometric LPs are equivalent. We now describe a different kind of equivalent LPs.

LPs with fractionally isomorphic matrices. Recall that a square matrix $X \geq$ 0 is doubly stochastic if its entries in each row and column sum up to 1 . We call two $m \times n$ matrices $M$ and $N$ fractionally isomorphic if there are doubly stochastic matrices $Y \in \mathbb{R}^{m \times m}$ and $Z \in \mathbb{R}^{n \times n}$ such that

$$
M Z=Y N \text { and } N Z^{t}=Y^{t} M \text {. }
$$

Grohe et al. [26, Eq. (5.1)-(5.2)] discuss similar definitions. Their purpose is to use fractional isomorphism and color refinement to reduce the dimension of linear equations and LPs. The meaning of (6) will be clear from the proof of Theorem 4.1 below.

Lemma 2.2. If $M$ and $N$ are fractionally isomorphic $m \times n$ matrices, then the linear programs $\operatorname{LP}\left(\mathbb{1}_{n}, M, \mathbb{1}_{m}\right.$,opt $)$ and $L P\left(\mathbb{1}_{n}, N, \mathbb{1}_{m}\right.$,opt $)$ are equivalent, where $\mathbb{1}_{n}$ denotes the $n$-dimensional all-ones vector.

Proof. Since the matrices $Y$ and $Z$ in (6) are doubly stochastic, $Y \mathbb{1}_{m}=\mathbb{1}_{m}$ and $\mathbb{1}_{n}^{t} Z=\mathbb{1}_{n}^{t}$. Along with the first equality in (6) $)$, these equalities imply that $L_{1} \leq L_{2}$. The reduction $L_{2} \leq L_{1}$ follows similarly from the second equality in (6) as $Y^{t}$ and $Z^{t}$ are doubly stochastic.

\section{The Weisfeiler-Leman algorithm: Notation and formal definitions}

We consider undirected graphs, possibly with colored vertices. The vertex set and the edge set of a graph $G$ are denoted by $V(G)$ and $E(G)$ respectively. For $\bar{x}=$ $\left(x_{1}, \ldots, x_{k}\right)$ in $V(G)^{k}$, let $\mathrm{WL}_{k}^{0}(G, \bar{x})$ be the $k \times k$ matrix $\left(m_{i, j}\right)$ with $m_{i, j}=1$ if $x_{i} x_{j} \in$ $E(G), m_{i, j}=2$ if $x_{i}=x_{j}$ and $m_{i, j}=0$ otherwise. We also augment $\mathrm{WL}_{k}^{0}(G, \bar{x})$ by the vector of the colors of $x_{1}, \ldots, x_{k}$ if the graph $G$ is vertex-colored. $\mathrm{WL}_{k}^{0}(G, \bar{x})$ encodes the ordered isomorphism type of $\bar{x}$ in $G$ and serves as an initial coloring of $V(G)^{k}$ for $k$-WL. In the $r^{t h}$ refinement round, $k$-WL computes a coloring $\mathrm{WL}_{k}^{r}(G, \cdot)$ of the Cartesian power $V(G)^{k}$ such that, if $\mathcal{P}_{r}$ is the color partition of $V(G)^{k}$ according to $\mathrm{WL}_{k}^{r}(G, \cdot)$, then $\mathcal{P}_{r+1}$ is finer than or equal to $\mathcal{P}_{r}$ for every $r \geq 0$. Specifically, $1-\mathrm{WL}$ 
computes $\mathrm{WL}_{1}^{r+1}(G, x)=\left(\mathrm{WL}_{1}^{r}(G, x),\left\{\left\{\mathrm{WL}_{1}^{r}(G, y): y \in N(x)\right\}\right)\right.$, where $N(x)$ is the neighborhood of $x$ and $\{\{\}$ denotes a multiset. If $k \geq 2, k$-WL refines the coloring by $\mathrm{WL}_{k}^{r+1}(G, x)=\left(\mathrm{WL}_{k}^{r}(G, \bar{x}),\left\{\left\{\left(\mathrm{WL}_{k}^{r}\left(G, \bar{x}_{1}^{u}\right), \ldots, \mathrm{WL}_{k}^{r}\left(G, \bar{x}_{k}^{u}\right): u \in V(G)\right\}\right)\right.\right.$, where $\bar{x}_{i}^{u}$ is the tuple $\left(x_{1}, \ldots, x_{i-1}, u, x_{i+1}, \ldots, x_{k}\right)$. If $G$ has $n$ vertices, the color partition $\mathcal{P}_{r}$ stabilizes in at most $n^{k}$ rounds. We define $\mathrm{WL}_{k}(G, \bar{x})=\mathrm{WL}_{k}^{n^{k}}(G, \bar{x})$ and $\mathrm{WL}_{k}(G)=\left\{\mathrm{WL}_{k}(G, \bar{x}): \bar{x} \in V(G)^{k}\right\}$. Now, $G \equiv_{k \text {-WL }} H$ if $\mathrm{WL}_{k}(G)=\mathrm{WL}_{k}(H)$.

The color partition of $V(G)$ according to $\mathrm{WL}_{1}(G, x)$ is equitable: for any color classes $C$ and $C^{\prime}$, each vertex in $C$ has the same number of neighbors in $C^{\prime}$. Moreover, if $G$ is vertex-colored, then the original colors of all vertices in each $C$ are the same. It is known [41, Theorem 6.5.1] that $G \equiv_{1-\mathrm{WL}} H$ exactly when $G$ and $H$ have a common equitable partition with the same neighborhood numbers in both graphs, after a suitable identification of vertex sets $V(G)$ and $V(H)$ (the coarsest such partition is actually the partition defined by the coloring $\left.\mathrm{WL}_{1}(G, \cdot)\right)$.

Let $G$ and $H$ be graphs with vertex set $\{1, \ldots, n\}$, and let $A$ and $B$ be the adjacency matrices of $G$ and $H$, respectively. Then $G$ and $H$ are isomorphic if and only if $A X=X B$ for some $n \times n$ permutation matrix $X$. The linear programming relaxation allows $X$ to be a doubly stochastic matrix. If such an $X$ exists, $G$ and $H$ are said to be fractionally isomorphic. If $G$ and $H$ are colored graphs with the same partition of the vertex set into color classes, then it is additionally required that $X_{u, v}=0$ whenever $u$ and $v$ are of different colors. Building on [42], it is shown by [39] that two graphs are indistinguishable by 1-WL if and only if they are fractionally isomorphic (see also [41, Theorem 6.5.1]).

\section{Getting started}

\subsection{Fractional Set Packing}

The Set Packing problem is to maximize the number of pairwise disjoint sets in a given family of sets $\mathcal{S}=\left\{S_{1}, \ldots, S_{n}\right\}$, where each $S_{j} \subset\{1, \ldots, m\}$. The maximum is called in combinatorics the matching number of the hypergraph $\mathcal{S}$ and denoted by $\nu(\mathcal{S})$. The fractional version of the matching number can be expressed as a linear program $L P(\mathcal{S})=L P\left(\mathbb{1}_{n}, M, \mathbb{1}_{m}, \max \right)$ where $M$ is the $m \times n$ incidence matrix of $\mathcal{S}$ :

$$
\begin{aligned}
\max \sum_{i=1}^{n} x_{i} & \text { under } \\
x_{i} & \geq 0 \text { for every } i \leq n \\
\sum_{i: S_{i} \ni j} x_{i} & \leq 1 \text { for every } j \leq m .
\end{aligned}
$$

The optimum value

$$
\nu_{f}(\mathcal{S})=\operatorname{val}(L P(\mathcal{S}))
$$

is called the fractional matching number of $\mathcal{S}$. 
Let $I(\mathcal{S})$ denote the incidence graph of $\mathcal{S}$. That is, $I(\mathcal{S})$ is the vertex-colored bipartite graph with biadjacency matrix $M$, where the bipartition of the vertex set is defined by $m$ red vertices and $n$ blue vertices. A red vertex $j$ is adjacent to a blue vertex $i$ if $j \in S_{i}$.

Theorem 4.1. Let $\mathcal{S}_{1}$ and $\mathcal{S}_{2}$ be two families each consisting of $n$ subsets of the set $\{1, \ldots, m\}$. If $I\left(\mathcal{S}_{1}\right) \equiv_{1-W L} I\left(\mathcal{S}_{2}\right)$, then $\nu_{f}\left(\mathcal{S}_{1}\right)=\nu_{f}\left(\mathcal{S}_{2}\right)$.

Proof. Denote the incidence matrices of $\mathcal{S}_{1}$ and $\mathcal{S}_{2}$ by $M$ and $N$ respectively. Then

$$
A_{1}=\left(\begin{array}{cc}
0 & M \\
M^{t} & 0
\end{array}\right) \text { and } A_{2}=\left(\begin{array}{cc}
0 & N \\
N^{t} & 0
\end{array}\right)
$$

are the adjacency matrices of $I\left(\mathcal{S}_{1}\right)$ and $I\left(\mathcal{S}_{2}\right)$ respectively. Since $I\left(\mathcal{S}_{1}\right)$ and $I\left(\mathcal{S}_{2}\right)$ are indistinguishable by color refinement, these graphs are fractionally isomorphic, that is, there is a doubly stochastic matrix $X$ such that

$$
A_{1} X=X A_{2}
$$

and $X_{u v}=0$ whenever $u$ and $v$ are from different vertex color classes. The latter condition means that $X$ is the direct sum of an $m \times m$ doubly stochastic matrix $Y$ and an $n \times n$ doubly stochastic matrix $Z$, that is,

$$
X=\left(\begin{array}{ll}
Y & 0 \\
0 & Z
\end{array}\right) \text {. }
$$

Therefore, Equality (7) reads

$$
\left(\begin{array}{cc}
0 & M \\
M^{t} & 0
\end{array}\right)\left(\begin{array}{ll}
Y & 0 \\
0 & Z
\end{array}\right)=\left(\begin{array}{cc}
Y & 0 \\
0 & Z
\end{array}\right)\left(\begin{array}{cc}
0 & N \\
N^{t} & 0
\end{array}\right)
$$

yielding

$$
M Z=Y N \text { and } M^{t} Y=Z N^{t},
$$

that is, the matrices $M$ and $N$ are fractionally isomorphic. Lemma 2.2 implies that $L P\left(\mathcal{S}_{1}\right)$ and $L P\left(\mathcal{S}_{2}\right)$ are equivalent. Therefore, these LPs have equal values by Theorem 2.1.

The dual version of $L P(\mathcal{S})$ is the following minimization problem:

$$
\begin{aligned}
& \min \sum_{j=1}^{m} y_{j} \text { under } \\
& y_{j} \geq 0 \text { for every } j \leq m, \\
& \sum_{j \in S_{i}} y_{j} \geq 1 \text { for every } i \leq n .
\end{aligned}
$$

This is an LP relaxation of the Hitting Set problem: Find a smallest set $Y \subset$ $\{1, \ldots, m\}$ (called a hitting set, cover, or transversal) having a non-empty intersection with each $S_{i}$. Denote the optimum value by $\tau_{f}(\mathcal{S})$ and note that $\tau_{f}(\mathcal{S})=\nu_{f}(\mathcal{S})$ by LP duality. 


\subsection{1-WL-invariance of the fractional domination number}

The closed neighborhood of a vertex $x$ is defined as $N[x]=N(x) \cup\{x\}$. A set $D \subseteq V(G)$ is dominating in $G$ if $V(G)=\bigcup_{x \in D} N[x]$. The domination number $\gamma(G)$ is the minimum cardinality of a dominating set in $G$.

As a warm-up example, consider the fractional Dominating Set problem, whose 1-WL-invariance was established in [40]:

$$
\begin{aligned}
\min \sum_{v \in V(G)} y_{v} & \text { under } \\
y_{v} & \geq 0 \text { for every } v \in V(G), \\
\sum_{v \in N[u]} y_{v} & \geq 1 \text { for every } u \in V(G) .
\end{aligned}
$$

The value of this LP is the fractional domination number $\gamma_{f}(G)$. We can see this as the fractional Hitting Set problem for $\mathcal{S}=\mathcal{S}_{G}$ consisting of the closed neighborhoods of all vertices in $G$. The incidence matrix $M$ of $\mathcal{S}_{G}$ and the adjacency matrix $A$ of the graph $G$ are related by the equality $M=A+I$. If $G \equiv_{1 \text {-wL }} H$, then $G$ and $H$ are fractionally isomorphic. That is, $A X=X B$ for a doubly stochastic $X$, where $B$ is the adjacency matrix of $H$. It follows that $M X=(A+I) X=X(B+I)=X N$, where $N$ is the incidence matrix of $\mathcal{S}_{H}$. Similarly, $X^{t} M=N X^{t}$. Therefore, $\gamma_{f}(G)=\gamma_{f}(H)$ by Lemma 2.2 and Theorem 2.1. This follows also from Theorem 4.1 as $I\left(\mathcal{S}_{G}\right) \equiv_{1-\mathrm{WL}} I\left(\mathcal{S}_{H}\right)$ and $\gamma_{f}(G)=\tau_{f}\left(\mathcal{S}_{G}\right)=\nu_{f}\left(\mathcal{S}_{G}\right)$ by LP duality.

\subsection{WL invariance through first-order interpretability}

As we have just seen, given an instance graph $G$ of the fractional Dominating Set problem, we can define an instance $\mathcal{S}_{G}$ of the fractional Hitting Set problem having the same LP value. The next definition formalizes a general setting that is applicable to essentially any logical formalism.

Definition 4.2. We say that an instance $\mathcal{S}_{G}$ of Fractional Set Packing or its dual version is definable over a graph $G$ with excess e if

$$
G \equiv_{(1+e)-\mathrm{WL}} H \Longrightarrow I\left(\mathcal{S}_{G}\right) \equiv_{1-\mathrm{WL}} I\left(\mathcal{S}_{H}\right) .
$$

This definition is very general. It includes settings where the incidence graph $I\left(\mathcal{S}_{G}\right)$ is first-order interpretable in the graph $G$ in the sense of [18, Chapter 12.3]. In other words, both the color predicate of $I\left(\mathcal{S}_{G}\right)$ (defining the red/blue bipartition of $\left.I\left(\mathcal{S}_{G}\right)\right)$ and the adjacency relation of $I\left(\mathcal{S}_{G}\right)$ are first-order expressible in terms of the adjacency relation of $G$, on $k$-tuples of vertices $V(G)^{k}$ for some $k$. The number $k$ is the width of the interpretation. In such a case, if there is a first-order sentence over $s$ variables that is true on $I\left(\mathcal{S}_{G}\right)$ but false on $I\left(\mathcal{S}_{H}\right)$, then there is a first-order sentence over $s k$ variables that is true on $G$ but false on $H$. Now, by the Cai-FürerImmerman result 9 , that two structures are $k$-WL-equivalent iff they are equivalent in the $(k+1)$-variable counting $\operatorname{logic} C^{k+1}$, we obtain the following corollary from Theorem 4.1. 
Corollary 4.3. Let $\pi_{f}$ be a fractional graph parameter such that $\pi_{f}(G)=\nu_{f}\left(\mathcal{S}_{G}\right)$, where $\mathcal{S}_{G}$ admits a first-order interpretation (possibly with counting quantifiers) of width $k$ in $G$. Then $\mathcal{S}_{G}$ is definable over $G$ with excess $2(k-1)$ and, consequently, $\pi_{f}$ is $(2 k-1)$-WL-invariant.

Remark 4.4. In order to obtain 1-WL-invariance via Theorem 4.1, we need definability with zero excess. Applying Corollary 4.3 for this purpose would require a first-order interpretation of width 1 , which may not always be possible. However, this is not the only way to get zero excess.

As an example (in a slightly general setting), consider $L P\left(\mathbb{1}_{n}, A^{2}, \mathbb{1}_{n}\right.$, opt) where $A$ is the adjacency matrix of $G$. As easily seen, if $G \equiv_{1-W L} H$, then there is a doubly stochastic $X$ such that $A^{2} X=A X B=X B^{2}$, and also $B^{2} X^{t}=X^{t} A^{2}$ by taking transpose. Therefore, the value of $L P\left(\mathbb{1}_{n}, A^{2}, \mathbb{1}_{n}\right.$, opt $)$ is 1 -WL-invariant 3 The logical route to show 1-WL-invariance via Corollary 4.3 is not feasible because the $(i, j)^{t h}$ entry of $A^{2}$ counts the number of 2 -walks between vertices $i$ and $j$, which cannot be captured by the logic $C^{2}$.

Another example where a combinatorial argument yields more than Corollary 4.3 is presented below.

\subsection{1-WL-invariance of the fractional matching number}

Recall that a set of edges $M \subseteq E(G)$ is a matching in a graph $G$ if every vertex of $G$ is incident to at most one edge from $M$. The matching number $\nu(G)$ is the maximum size of a matching in $G$. Note that this terminology and notation agrees with Section 4.1 when graphs are considered hypergraphs with hyperedges of size 2 . The fractional Matching Problem is defined by the LP

$$
\begin{aligned}
& \max \sum_{u v \in E(G)} x_{u v} \text { under } \\
& x_{u v} \geq 0 \text { for every } u v \in E(G), \\
& \sum_{v \in N(u)} x_{u v} \leq 1 \text { for every } u \in V(G),
\end{aligned}
$$

whose value is the fractional matching number $\nu_{f}(G)$. The above LP is exactly the linear program $L P\left(\mathcal{S}_{G}\right)$ for the instance $\mathcal{S}_{G}=E(G)$ of Fractional Set Packing formed by the edges of $G$ as 2-element subsets of $V(G)$, that is, $\nu_{f}(G)=\nu_{f}\left(\mathcal{S}_{G}\right)$. A first-order interpretation of the incidence graph $\mathcal{S}_{G}$ in the input graph $G$ of width 1 is clearly impossible. Moreover, an interpretation of width 2 can only give 3-WLinvariance by Corollary 4.3. Nevertheless, we can directly show that $\mathcal{S}_{G}$ is definable over $G$ with zero excess.

Theorem 4.5. The fractional matching number is 1-WL-invariant.

\footnotetext{
${ }^{3}$ Indeed, as observed by Rubalcaba [40, this holds for any polynomial in $A$
} 
Proof. Given $G \equiv_{1 \text {-wL }} H$, we have to prove that $\nu_{f}(G)=\nu_{f}(H)$ or, equivalently, $\nu_{f}\left(\mathcal{S}_{G}\right)=\nu_{f}\left(\mathcal{S}_{H}\right)$ where $\mathcal{S}_{G}$ is as defined above. By Theorem 4.1, it suffices to show that $I\left(\mathcal{S}_{G}\right) \equiv_{1 \text {-WL }} I\left(\mathcal{S}_{H}\right)$. To this end, we construct a common equitable partition of $I\left(\mathcal{S}_{G}\right)$ and $I\left(\mathcal{S}_{H}\right)$, appropriately identifying their vertex sets. Recall that $V\left(I\left(\mathcal{S}_{G}\right)\right)=V(G) \cup E(G)$ and a red vertex $x \in V(G)$ is adjacent to a blue vertex $e \in E(G)$ if $x \in e$.

For $x \in V(G)$, let $c_{G}(x)=\mathrm{WL}_{1}(G, x)$ and define $c_{H}$ on $V(H)$ similarly. First, we identify $V(G)$ and $V(H)$ (i.e., the red parts of the two incidence graphs) so that $c_{G}(x)=c_{H}(x)$ for every $x$ in $V(G)=V(H)$, which is possible because 1-WLequivalent graphs have the same color palette after color refinement. The color classes of $c_{G}$ now form a common equitable partition of $G$ and $H$.

Next, extend the coloring $c_{G}$ to $E(G)$ (the blue part of $\left.I\left(\mathcal{S}_{G}\right)\right)$ by $c_{G}(\{x, y\})=$ $\left\{c_{G}(x), c_{G}(y)\right\}$, and similarly extend $c_{H}$ to $E(H)$. Denote the color class of $c_{G}$ containing $\{x, y\}$ by $C_{G}(\{x, y\})$, the color class containing $x$ by $C_{G}(x)$ etc. Note that $\left|C_{G}(\{x, y\})\right|$ is equal to the number of edges in $G$ between $C_{G}(x)$ and $C_{G}(y)$ (or the number of edges within $C_{G}(x)$ if $c_{G}(x)=c_{G}(y)$ ). Since $\left\{C_{G}(x)\right\}_{x \in V(G)}$ is a common equitable partition of $G$ and $H$, we have $\left|C_{G}(\{x, y\})\right|=\left|C_{H}\left(\left\{x^{\prime}, y^{\prime}\right\}\right)\right|$ whenever $c_{G}(\{x, y\})=c_{H}\left(\left\{x^{\prime}, y^{\prime}\right\}\right)$ (note that for any edge $\{x, y\}$ of $G$ there is an edge $\left\{x^{\prime}, y^{\prime}\right\}$ of the same color in $H$ and vice versa). This allows us to identify $E(G)$ and $E(H)$ so that $c_{G}(e)=c_{H}(e)$ for every $e$ in $E(G)=E(H)$.

Now, consider the partition of $V(G) \cup E(G)$ into the color classes of $c_{G}$ (or the same in terms of $H)$ and verify that this is a common equitable partition of $I\left(\mathcal{S}_{G}\right)$ and $I\left(\mathcal{S}_{H}\right)$. Indeed, let $C$ and $D$ be color classes of $c_{G}$ (hence, also of $c_{H}$ ). It is enough to consider the case $C \subseteq V(G)$ and $D \subseteq E(G)$ because otherwise $C$ and $D$ are in the same part of the incidence graph and there is no edge in between. Consider an arbitrary vertex $x \in C$ and an arbitrary edge $e \in D$. Let $e=\{u, v\}$. If neither $u$ nor $v$ is in $C$, then there is no edge between $C$ and $D$ in both $I\left(\mathcal{S}_{G}\right)$ and $I\left(\mathcal{S}_{H}\right)$. Otherwise, suppose that $u \in C$ and denote $C^{\prime}=C_{G}(v)$ (it is not excluded that $\left.C^{\prime}=C\right)$. Clearly, the vertex $x$ has exactly as many $D$-neighbors in $I\left(\mathcal{S}_{G}\right)$ as it has $C^{\prime}$-neighbors in $G$. This number depends only on $C$ and $C^{\prime}$ or, equivalently, only on $C$ and $D$, and is the same if counted for $I\left(\mathcal{S}_{H}\right)$.

On the other hand, $e$ has exactly one $C$-neighbor, $u$, in $I\left(\mathcal{S}_{G}\right)$ and $I\left(\mathcal{S}_{H}\right)$ if $C^{\prime} \neq C$ and exactly two $C$-neighbors, $u$ and $v$, if $C^{\prime}=C$. What is the case depends only on $D$ and $C$, and is the same in $I\left(\mathcal{S}_{G}\right)$ and $I\left(\mathcal{S}_{H}\right)$. Thus, we do have a common equitable partition of $I\left(\mathcal{S}_{G}\right)$ and $I\left(\mathcal{S}_{H}\right)$.

The fractional matching number is precisely the fractional $K_{2}$-packing number, and we generalize Theorem 4.5 to fractional $F$-packing numbers in Section 5 . In particular, there we will establish 2-WL-invariance of Fractional Triangle Packing. The approach we used in the proof of Theorem 4.5 works as well for edge-disjoint packing, which we demonstrate in the next subsection. 


\subsection{2-WL-invariance of Fractional Edge-Disjoint Triangle Pack- ing}

Given a graph $G$, let $T(G)$ denote the family of all sets $\left\{e_{1}, e_{2}, e_{3}\right\}$ consisting of the edges of a triangle subgraph in $G$. We regard $T(G)$ as a family $\mathcal{S}_{G}$ of subsets of the edge set $E(G)$. The optimum value of Set Packing Problem on $\mathcal{S}_{G}$, which we denote by $\rho^{K_{3}}(G)$, is equal to the maximum number of edge-disjoint triangles in $G$. Let $\rho_{f}^{K_{3}}(G)=\nu_{f}\left(\mathcal{S}_{G}\right)$ be the corresponding fractional parameter.

Theorem 4.6. The fractional edge-disjoint triangle packing number $\rho_{f}^{K_{3}}$ is 2-WLinvariant.

Proof. Given a graph $G$, we consider the coloring $c_{G}$ of $E(G) \cup T(G)$ defined by $c_{G}(\{x, y\})=\left\{\mathrm{WL}_{2}(G, x, y), \mathrm{WL}_{2}(G, y, x)\right\}$ on $E(G)$ and $c_{G}\left(\left\{e_{1}, e_{2}, e_{3}\right\}\right)=\left\{\left\{c_{G}\left(e_{1}\right)\right.\right.$, $\left.\left.c_{G}\left(e_{2}\right), c_{G}\left(e_{3}\right)\right\}\right\}$ on $T(G)$. As in the proof of Theorem 4.5, the upper case notation $C_{G}(s)$ will be used to denote the color class containing $s \in E(G) \cup T(G)$.

Suppose that $G \equiv_{2 \text {-WL }} H$. This condition implies that we can identify the sets $E(G)$ and $E(H)$ so that $c_{G}(e)=c_{H}(e)$ for every $e$ in $E(G)=E(H)$. Moreover, the 2-WL-equivalence of $G$ and $H$ implies that for any $t$ in $T(G)$ there is $t^{\prime}$ of the same color in $T(H)$ and vice versa. What is more, for any $t \in T(G)$ and $t^{\prime} \in T(H)$ with $c_{G}(t)=c_{H}\left(t^{\prime}\right)$ we have $\left|C_{G}(t)\right|=\left|C_{H}\left(t^{\prime}\right)\right|$. This allows us to identify $T(G)$ and $T(H)$ so that $c_{G}(t)=c_{H}(t)$ for every $t$ in $T(G)=T(H)$. As in the proof of Theorem 4.5, it suffices to argue that $\left\{C_{G}(w)\right\}_{w \in E(G) \cup T(G)}$ is a common equitable partition of the incidence graphs $I\left(\mathcal{S}_{G}\right)$ and $I\left(\mathcal{S}_{H}\right)$. The equality $\rho_{f}^{K_{3}}(G)=\rho_{f}^{K_{3}}(H)$ will then follow by Theorem 4.1 .

Let $C \subseteq E(G)$ and $D \subseteq T(G)$ be color classes of $c_{G}$ (hence, also of $c_{H}$ ). Consider an arbitrary triangle $t=\left\{e_{1}, e_{2}, e_{3}\right\}$ in $D$. If none of $e_{i}$ belongs to $C$, then there is no edge between $C$ and $D$ in both $I\left(\mathcal{S}_{G}\right)$ and $I\left(\mathcal{S}_{H}\right)$. Suppose that $e_{1} \in C$ and denote $C^{\prime}=C_{G}\left(e_{2}\right)$ and $C^{\prime \prime}=C_{G}\left(e_{3}\right)$ (it is not excluded that some of the classes $C, C^{\prime}$, and $C^{\prime \prime}$ coincide).

Denote the vertices of $t$ by $u, v, w$ and suppose that $e_{1}=\{u, v\}$. Consider an arbitrary edge $e=\{x, y\}$ in $C$. Let us count the number of $D$-neighbors that $e$ has in $I\left(\mathcal{S}_{G}\right)$, that is, the number of triangles in $G$ with one edge $e$ and two other edges $e^{\prime} \in C^{\prime}$ and $e^{\prime \prime} \in C^{\prime \prime}$. This number is equal to the number of vertices $z$ such that $\left(\mathrm{WL}_{2}(G, x, z), \mathrm{WL}_{2}(G, z, y)\right)$ is one of the 8 pairs in $\left(c_{G}(\{u, w\}) \times c_{G}(\{v, w\})\right) \cup$ $\left(c_{G}(\{v, w\}) \times c_{G}(\{u, w\})\right)$, like $\left(\mathrm{WL}_{2}(G, w, v), \mathrm{WL}_{2}(G, u, w)\right)$ (some of these pairs can coincide). Since the partition of $V(G)^{2}$ by the coloring $\mathrm{WL}_{2}(G, \cdot, \cdot)$ is not further refined by $2-\mathrm{WL}$, this number does not depend on the choice of $e$ in $C$, depending only on $C$ and $D$. We obtain the same number also while counting the $D$-neighbors of $e$ in $I\left(\mathcal{S}_{H}\right)$.

On the other hand, $t$ has exactly one neighbor $e_{1}$ in $C$ if $C$ differs from both $C^{\prime}$ and $C^{\prime \prime}$, exactly two $C$-neighbors if $C$ coincides with exactly one of $C^{\prime}$ and $C^{\prime \prime}$, and exactly three $C$-neighbors $e_{1}, e_{2}$, and $e_{3}$ if $C=C^{\prime}=C^{\prime \prime}$. Which of the three possibilities occurs depends only on $D$ and $C$, and is the same in $I\left(\mathcal{S}_{G}\right)$ and 
$I\left(\mathcal{S}_{H}\right)$. This completes our analysis, showing that we really have a common equitable partition of $I\left(\mathcal{S}_{G}\right)$ and $I\left(\mathcal{S}_{H}\right)$.

Note that 2-WL-invariance in Theorem 4.6 is optimal. Indeed, for the 1-WLequivalent graphs $2 C_{3}$ and $C_{6}$ we have $\rho_{f}^{K_{3}}\left(2 C_{3}\right)=2$ while $\rho_{f}^{K_{3}}\left(C_{6}\right)=0$.

\section{The $F$-packing number}

For graphs $F$ and $G$, let $\operatorname{Sub}(F, G)$ denote the set of all subgraphs $S$ of $G$ that are isomorphic to $F$. An F-packing of $G$ is a set $P \subseteq \operatorname{Sub}(F, G)$ where all subgraphs are vertex disjoint. The $F$-packing number $\pi^{F}(G)$ is the maximum size of an $F$ packing $P$ of $G$. Let $\mathcal{S}_{F, G}=\{V(S): S \in \operatorname{Sub}(F, G)\}$. Note that $\pi^{F}(G)=\nu\left(\mathcal{S}_{F, G}\right)$, the matching number of the hypergraph $\mathcal{S}_{F, G}$. The fractional F-packing number of $G$ is defined by $\pi_{f}^{F}=\nu_{f}\left(\mathcal{S}_{F, G}\right)$, where $\nu_{f}$ is the fractional matching number of a hypergraph as introduced in Section 4.1 .

The following parameter plays a key role in our approach to estimating the WL dimension of $\pi_{f}^{F}$. We define the homomorphism-hereditary treewidth of a graph $F$, denoted by $h t w(F)$, as the maximum treewidth $t w\left(F^{\prime}\right)$ over all homomorphic images $F^{\prime}$ of $F$, i.e., over all $F^{\prime}$ such that there is a homomorphism $h$ from $F$ to $F^{\prime}$ that is vertex and edge surjective.

For a colored graph $S$, let $S^{\circ}$ denote the underlying uncolored version of $S$.

We begin with an important technical lemma. In what follows, $F$ is an uncolored graph and $G$ is a graph endowed with a coloring $c: V(G) \rightarrow\{1, \ldots, r\}$. For a subgraph $S$ of $G$, we define its color type as the multiset $\mu(S)=\{c(u): u \in V(S)\}$. For a given color type $\mu$, we write $\operatorname{Sub}(\mu, F, G)$ to denote the set of all subgraphs $S$ of $G$ such that $S^{\circ}$ is isomorphic to $F$ and $\mu(S)=\mu$. The number of subgraphs $S \in \operatorname{Sub}(\mu, F, G)$ containing a vertex $x$ is denoted by $\operatorname{sub}(x, \mu, F, G)$.

Given $x \in V(G)$, we set $\mathrm{WL}_{k}(G, x)=\mathrm{WL}_{k}(G, \bar{x})$ where $\bar{x}$ is the $k$-tuple whose all elements are equal to $x$. Dvořák [17] proves that, for each graph $F$ of treewidth $k$, the number of homomorphisms from $F$ to a graph $G$ is $k$-WL-invariant. In particular [17. Lemma 4], if $\mathrm{WL}_{k}(G, x)=\mathrm{WL}_{k}(H, y)$ and $F$ is a graph of treewidth at most $k$ with a designated vertex $z$, then the number of homomorphisms from $F$ to $G$ taking $z$ to $x$ is equal to the number of homomorphisms from $F$ to $H$ taking $z$ to $y$. The classical result by Lovász [36, Section 5.2.3] shows the number of subgraphs of a graph $G$ isomorphic to $F$ is determined by the numbers of homomorphisms from $F^{\prime}$ to $G$, where $F^{\prime}$ ranges over homomorphic images of $F$. Combined with Dvořák's result, this implies the following fact (see [21] for some details).

Lemma 5.1. If $h t w(F) \leq k$, then the count $\operatorname{sub}(x, \mu, F, G)$ is determined by the quadruple $F, \mu, \mathrm{WL}_{k}(G, x)$, and $\mathrm{WL}_{k}(G)$.

Note that, if $k \geq 2$, then $\mathrm{WL}_{k}(G) \neq \mathrm{WL}_{k}(H)$ even implies that $\mathrm{WL}_{k}(G) \cap \mathrm{WL}_{k}(H)=$ $\emptyset$. This means that the stable color $\mathrm{WL}_{k}(G, x)$ of any single vertex $x$ determines the color palette $\mathrm{WL}_{k}(G)$. Thus, for $k \geq 2$, Lemma 5.1 says that $\operatorname{sub}(x, \mu, F, G)$ is determined by the triple $F, \mu$, and $\mathrm{WL}_{k}(G, x)$. 
Denote the incidence graph of the hypergraph $\mathcal{S}_{F, G^{\circ}}$ by $G^{F}$. Recall that $G^{F}$ is a colored graph with two color classes $V(G)$ (red) and $\operatorname{Sub}\left(F, G^{\circ}\right)$ (blue) where a red vertex $x$ is adjacent to a blue vertex $S$ if $x \in V(S)$.

Lemma 5.2. Let $G$ be a graph with vertex coloring $c$ and $\mathcal{P}=\left\{C_{1}, \ldots, C_{r}\right\}$ be the corresponding color partition of $V(G)$. For a graph $F$, suppose that the counts $\operatorname{sub}(x, \mu, F, G)$ only depend on $(c(x), \mu, F)$. Then the partition $\mathcal{P}$ can be extended to an equitable partition $\mathcal{P}^{F}=\left\{C_{1}, \ldots, C_{r+s}\right\}$ of $G^{F}$. Specifically, if $\mu_{1}, \ldots, \mu_{s}$ are all color types $\mu$ with $\operatorname{Sub}(\mu, F, G) \neq \emptyset$, then $C_{r+i}=\left\{S^{\circ}: S \in \operatorname{Sub}\left(\mu_{i}, F, G\right)\right\}$ for $i=1, \ldots, s$.

Proof. For each pair $x, y$ of vertices in each $C_{i}$, we need to show that $x$ and $y$ have the same number of neighbors in each $C_{j}$. If $i, j \leq r$ or $i, j>r$, then between $C_{i}$ and $C_{j}$ there is no edge at all. If $i \leq r$ and $j>r$, then $\operatorname{sub}\left(x, \mu_{j-r}, F, G\right)=\operatorname{sub}\left(y, \mu_{j-r}, F, G\right)$ as $c(x)=c(y)=i$. Since $\operatorname{sub}\left(x, \mu_{j-r}, F, G\right)$ is exactly the number of neighbors of $x$ in $C_{j}$, the claim follows also in this case. Finally, for $i>r$ and $j \leq r$ the color type $\mu_{i-r}$ contains the color corresponding to $C_{j}$ with a certain multiplicity $m_{j}$ which for any vertex $x \in C_{i}$ coincides with the number of its neighbors in $C_{j}$.

Theorem 5.3. If htw $(F) \leq k$, then $\pi_{f}^{F}$ is $k$-WL-invariant.

Proof. Let $G$ and $H$ be two $k$-WL-equivalent graphs. Color the vertices of $G$ and $H$ with their stable $k$-WL vertex colors, that is, assign each vertex $x \in V(G)$ color $c(x)=\mathrm{WL}_{k}(G, x)$ and each vertex $x \in V(H)$ color $c(x)=\mathrm{WL}_{k}(H, x)$. Since $\mathrm{WL}_{k}(G)=\mathrm{WL}_{k}(H)$, we can identify the vertex sets $V(G)$ and $V(H)$ so that each vertex $x$ has the same color $c(x)$ in both graphs. Let $\mathcal{P}=\left\{C_{1}, \ldots, C_{r}\right\}$ be the corresponding color partition of $V(G)=V(H)$.

Moreover, it follows from Lemma 5.1 that the counts $\operatorname{sub}(x, \mu, F, G)$ and $\operatorname{sub}(x, \mu$, $F, H)$ coincide and are uniform within each vertex class $C_{i}$. This implies, in particular, that the color types $\mu_{1}, \ldots, \mu_{s}$ of subgraphs $S$ with $S^{\circ} \cong F$ appearing in $G$ and in $H$ are the same. Let $\mathcal{P}_{G}^{F}=\left\{C_{1}, \ldots, C_{r+s}\right\}$ and $\mathcal{P}_{H}^{F}=\left\{C_{1}^{\prime}, \ldots, C_{r+s}^{\prime}\right\}$ be the equitable partitions of $G^{F}$ and $H^{F}$ given by Lemma 5.2. Recall that $x$ has $\operatorname{sub}\left(x, \mu_{i}, F, G\right)=\operatorname{sub}\left(x, \mu_{i}, F, H\right)$ neighbors in $C_{r+i}$ in the graph $G^{F}$ and the same number of neighbors in $C_{r+i}^{\prime}$ in the graph $H^{F}$. On the other hand, any two subgraphs $S \in C_{r+i}$ and $S^{\prime} \in C_{r+i}^{\prime}$ have the same color type $\mu_{i}$ and, therefore, equally many neighbors in each $C_{j}, j \leq r$, in $G^{F}$ and in $H^{F}$. We conclude from here that $\left|C_{r+i}\right|=\left|C_{r+i}^{\prime}\right|$ for every $i \leq s$. Therefore, $\operatorname{Sub}(F, G)$ and $\operatorname{Sub}(F, H)$ can be identified so that $C_{r+i}=C_{r+i}^{\prime}$ for every $i \leq s$, and $\mathcal{P}^{F}=\mathcal{P}_{G}^{F}=\mathcal{P}_{H}^{F}$ becomes a common equitable partition of $G^{F}$ and $H^{F}$.

The existence of a common equitable partition implies that $G^{F}$ and $H^{F}$ are 1-WL-equivalent. Applying Theorem 4.1, we conclude that $\pi_{f}^{F}(G)=\pi_{f}^{F}(H)$.

Remark 5.4. If subgraphs in $P \subseteq \operatorname{Sub}(F, G)$ are allowed to share vertices but required to be edge disjoint, we call $P$ an edge disjoint $F$-packing of $G$. The edge disjoint $F$-packing number $\rho^{F}$ is defined as the maximum size of such $P$. Redefine $\mathcal{S}_{F, G}$ by $\mathcal{S}_{F, G}=\{E(S): S \in \operatorname{Sub}(F, G)\}$, which now becomes a hypergraph on the 
set $E(G)$. Now, $\rho^{F}(G)=\nu\left(\mathcal{S}_{F, G}\right)$, and we also define the fractional edge-disjoint $F$-packing number of $G$ by $\rho_{f}^{F}=\nu_{f}\left(\mathcal{S}_{F, G}\right)$. Similarly to Theorem 5.3, if $h t w(F) \leq k$, then $\rho_{f}^{F}$ is $k$-WL-invariant. Basically the same argument works out, and we here only comment on some proof details (cf. also the proof of Theorem 4.6).

We set $\mathrm{WL}_{k}(G, x, y)=\mathrm{WL}_{k}(G, \bar{z})$ where the pair $(x, y)$ is extended to the $k$ tuple $\bar{z}=(x, y, \ldots, y)$ if $k>2$. If $k=1$, we define $\mathrm{WL}_{1}(G, x, y)=\left(\mathrm{WL}_{1}(G, x)\right.$, $\left.\mathrm{WL}_{1}(G, y)\right)$. For an edge $e=\{x, y\}$ of $G$, denote

$$
c(e)=\left\{\mathrm{WL}_{k}(G, x, y), \mathrm{WL}_{k}(G, y, x)\right\} .
$$

For a subgraph $S$ of $G$, we adapt the notion of the color type of $S$, now defining it by $\mu(S)=\{\{c(e): e \in E(S)\}$.

Let $\operatorname{sub}(e, \mu, F, G)$ denote the number of subgraphs $S \in \operatorname{Sub}(F, G)$ having color type $\mu$ and containing an edge $e \in E(G)$. An analog of Lemma 5.1 says that, for a pair of adjacent vertices $x$ and $y$, the count $\operatorname{sub}(\{x, y\}, \mu, F, G)$ is determined by the tuple $\left.\operatorname{WLL}_{k}(G, x, y), \mu, F\right)$ (note that $\mathrm{WL}_{k}(G, x, y)$ determines $\mathrm{WL}_{k}(G, y, x)$ and vice versa). Lemma 5.2 has an analog for the edge coloring $c$ introduced above.

\section{Invariance ratio and integrality gap}

\subsection{Edge-disjoint triangle packing: Invariance ratio}

We now turn back to the edge-disjoint triangle packing number $\rho^{K_{3}}$ and its fractional version $\rho_{f}^{K_{3}}$, defined in Section 4.5, Let

$$
I G^{K_{3}}=\sup _{G} \frac{\rho_{f}^{K_{3}}(G)}{\rho^{K_{3}}(G)}
$$

where the supremum is taken over all graphs containing at least one triangle, be the integrality gap of $\rho_{f}^{K_{3}}$. We define the invariance ratio of $\rho^{K_{3}}$ by

$$
I R^{K_{3}}=\sup _{G, H}\left|\frac{\rho^{K_{3}}(G)}{\rho^{K_{3}}(H)}\right|
$$

where the supremum is taken over all pairs of 2-WL-equivalent graphs $G$ and $H$ containing at least one triangle. If $G$ and $H$ are such graphs, then, by Theorem 4.6,

$$
\rho^{K_{3}}(G) \leq \rho_{f}^{K_{3}}(G)=\rho_{f}^{K_{3}}(H) \leq I G^{K_{3}} \cdot \rho^{K_{3}}(H),
$$

which implies the relationship

$$
I G^{K_{3}} \geq I R^{K_{3}} .
$$

Theorem 6.1. $I G^{K_{3}}=I R^{K_{3}}=2$. 
(a)

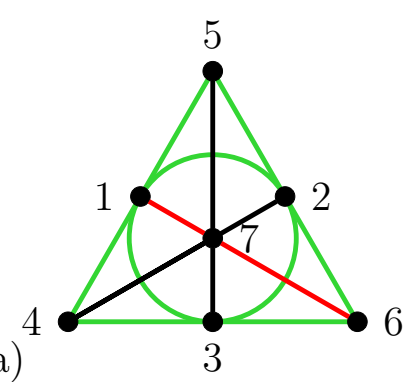

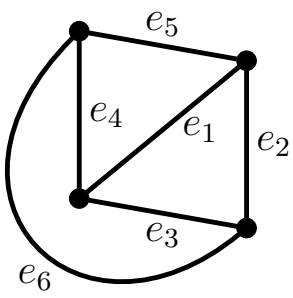

(b)

Figure 1: (a) Fano plane (b) The only constellation of triangles consistent with the green hyperedges of the Fano plane. Adding a triangle for the red hyperedge is impossible.

Proof. We begin with proving a lower bound $I G^{K_{3}} \leq 2$. In Section 4.1, we discussed the fractional matching number $\nu_{f}(\mathcal{S})$ of a hypergraph $\mathcal{S}$. Füredi [22] proved tight bounds for the integrality gap of this parameter. In particular, if a 3-uniform hypergraph $\mathcal{S}$ does not contain any set of seven hyperedges forming the Fano plane, then $\nu_{f}(\mathcal{S}) / \nu(\mathcal{S}) \leq 2$. Recall that a hypergraph is $r$-uniform if each hyperedge consists of $r$ vertices. The Fano plane is the 3-uniform hypergraph with 7 vertices and 7 hyperedges shown in Figure 1(a). Let $G$ be an arbitrary graph, and $\mathcal{S}_{G}$ be the hypergraph we associated with $G$ in Section 4.5. Recall that $\rho_{f}^{K_{3}}(G)=\nu_{f}\left(\mathcal{S}_{G}\right)$. In order to prove the bound $I G^{K_{3}} \leq 2$, it suffices to check that $\mathcal{S}_{G}$ does not contain any copy of the Fano plane.

Indeed, assume that $F$ is a copy of the Fano plane in $\mathcal{S}_{G}$. Enumerate the vertices of $F$ as in Figure 1(a). Each vertex $i$ of $F$ is an edge of $G$, which we denote by $e_{i}$. Each hyperedge of $F$ consists of the edges of a triangle in $G$. Thus, the triangles of $G$ corresponding to the hyperedges $\{1,2,3\}$ and $\{1,4,5\}$ share an edge $e_{1}$; see Figure 1(b). The edge $e_{6}$ must, therefore, connect the two vertices of these triangles not incident to $e_{1}$. Consequently, $e_{1}$ and $e_{6}$ are not adjacent, contradicting the fact that they belong to the hyperedge $\{1,6,7\}$.

We complete the proof by showing that $I R^{K_{3}} \geq 2$. Denote the Shrikhande and the $4 \times 4$ rook's graphs by $S$ and $R$ respectively. Both have vertex set $\mathbb{Z}_{4} \times \mathbb{Z}_{4}$, and $(i, j)$ and $\left(i^{\prime}, j^{\prime}\right)$ are adjacent in $S$ if $\left(i=i^{\prime}\right.$ and $\left.j^{\prime}=j+1\right)$ or $\left(j=j^{\prime}\right.$ and $\left.i^{\prime}=i+1\right)$ or $\left(i^{\prime}=i+1\right.$ and $\left.j^{\prime}=j+1\right)$, where equality is in $\mathbb{Z}_{4}$, while they are adjacent in $R$ if $i=i^{\prime}$ (row 4-clique) or $j=j^{\prime}$ (column 4-clique). The Shrikhande graph is completely decomposable into edge-disjoint triangles $\{(i, j),(i+1, j),(i+1, j+1)\}$ and, hence, $\rho^{K_{3}}(S)=16$. On the other hand, in $R$ the edges of each $K_{3}$ all belong to the same row or column 4-clique, and the rest of the edges in this row/column correspond to a star. Since a packing can take at most one $K_{3}$ from each row/column, we have $\rho^{K_{3}}(R)=8$. It remains to note that both $S$ and $R$ are strongly regular graphs with the same parameters $(16,6,2,2)$. Therefore, $S \equiv_{2 \text {-WL }} R$. 


\subsection{Edge-disjoint triangle packing: Invariance difference}

Denote the number of vertices in a graph $G$ by $v(G)$. Let

$$
A I G^{K_{3}}(n)=\max _{G: v(G)=n}\left(\rho_{f}^{K_{3}}(G)-\rho^{K_{3}}(G)\right)
$$

be the additive integrality gap of $\rho_{f}^{K_{3}}$. Haxell and Rödl [27] proved that

$$
A I G^{K_{3}}(n)=o\left(n^{2}\right)
$$

which gives a PTAS for $\rho^{K_{3}}$ on dense enough graphs. On the other hand, Yuster [44] showed that $A I G^{K_{3}}(n)=\Omega\left(n^{1.5}\right)$, and it is open whether this lower bound is tight. Define the invariance difference of $\rho^{K_{3}}$ as the function

$$
I D^{K_{3}}(n)=\max \left|\rho^{K_{3}}(G)-\rho^{K_{3}}(H)\right|
$$

where the maximum is taken over 2-WL-equivalent $n$-vertex graphs $G$ and $H$. As follows from Theorem 4.6, $I D^{K_{3}}(n)$ provides a lower bound for the additive integrality gap of $\rho_{f}^{K_{3}}$, namely

$$
A I G^{K_{3}}(n) \geq \frac{1}{2} I D^{K_{3}}(n) .
$$

In this respect, it would be interesting to determine the asymptotics of $I D^{K_{3}}(n)$ and to investigate how tight the relation (8) is. The following result is a step towards this goal.

Theorem 6.2. $I D^{K_{3}}(n)=\Omega\left(n^{1.25}\right)$.

The proof uses two lemmas, which we state and prove now. The tensor product $G \times G^{\prime}$ of graphs $G$ and $G^{\prime}$ is the graph on the vertex set $V(G) \times V\left(G^{\prime}\right)$ with vertices $\left(u, u^{\prime}\right)$ and $\left(v, v^{\prime}\right)$ adjacent if $u$ and $v$ are adjacent in $G$ and $u^{\prime}$ and $v^{\prime}$ are adjacent in $G^{\prime}$.

Lemma 6.3. If $G \equiv_{2 \text {-WL }} H$ and $G^{\prime} \equiv_{2 \text {-WL }} H^{\prime}$, then $G \times G^{\prime} \equiv_{2 \text {-WL }} H \times H^{\prime}$.

Proof. We use the fact 28 that $G \equiv_{2 \text {-wL }} H$ if and only if Duplicator has a winning strategy in the 3-pebble Hella's bijection game on $G$ and $H$. The game is played by two players, Spoiler and Duplicator. There are three pairwise distinct pebbles $p_{1}, p_{2}, p_{3}$, each given in duplicate. In one round of the game, Spoiler puts one of the pebbles $p_{i}$ on a vertex in $G$ and its copy on a vertex in $H$. When $p_{i}$ is on the board, $x_{i}$ denotes the vertex pebbled by $p_{i}$ in $G$, and $y_{i}$ denotes the vertex pebbled by the copy of $p_{i}$ in $H$. Specifically, a round is played as follows:

- Spoiler chooses $i \in\{1,2,3\}$;

- Duplicator responds with a bijection $f: V(G) \rightarrow V(H)$ obeying the condition that $f\left(x_{j}\right)=y_{j}$ for all $j \neq i$ such that $p_{j}$ is on the board; 
- Spoiler chooses a vertex $x$ in $G$ and puts $p_{i}$ on $x$ and its copy on $f(x)$ (this move reassigns $x_{i}$ to vertex $x$ and $y_{i}$ to vertex $\left.f(x)\right)$.

Duplicator wins if she manages to keep the map $x_{i} \mapsto y_{i}$ a partial isomorphism during the play; otherwise the winner is Spoiler.

The 2-WL-equivalence of $G$ and $H$ and of $G^{\prime}$ and $H^{\prime}$ implies that Duplicator has a winning strategy in the bijection game on $G$ and $H$ and on $G^{\prime}$ and $H^{\prime}$. She can combine these strategies to win also the game on $G \times G^{\prime}$ and $H \times H^{\prime}$ by regarding it as simultaneous play of a game on $G$ and $H$ and a game on $G^{\prime}$ and $H^{\prime}$. Whenever $\bar{x}_{i}=\left(x_{i}, x_{i}^{\prime}\right)$ and $\bar{y}_{i}=\left(y_{i}, y_{i}^{\prime}\right)$ are pebbled in $G \times G^{\prime}$ and $H \times H^{\prime}$, Duplicator assumes that $x_{i}$ and $y_{i}$ are pebbled in the game on $G$ and $H$ while $x_{i}^{\prime}$ and $y_{i}^{\prime}$ are pebbled in the game on $G^{\prime}$ and $H^{\prime}$. Given two bijections $f$ and $f^{\prime}$ for these games, she provides Spoiler with the bijection $\bar{f}$ defined by $\bar{f}\left(u, u^{\prime}\right)=\left(f(u), f^{\prime}\left(u^{\prime}\right)\right)$. Since $f$ and $f^{\prime}$ ensure partial isomorphisms between $G$ and $H$ and between $G^{\prime}$ and $H^{\prime}$, the bijection $\bar{f}$ maintains a partial isomorphism between $G \times G^{\prime}$ and $H \times H^{\prime}$. The existence of a winning strategy implies that the product graphs are 2-WL-equivalent.

We say that a graph $G$ is $K_{3}$-decomposable if there is an edge-disjoint triangle packing covering all edges of $G$, that is, $\rho^{K_{3}}(G)=e(G) / 3$.

Lemma 6.4. If both $G$ and $H$ are $K_{3}$-decomposable, then $G \times H$ is $K_{3}$-decomposable too.

Proof. Note first that the claim is true for $G=H=K_{3}$ because every edge in $K_{3} \times K_{3}$ has a unique extension to a triangle.

Let $T_{G}$ be a complete edge-disjoint triangle packing in $G$, and $T_{H}$ be a complete edge-disjoint triangle packing in $H$. The set of all possible products $t \times t^{\prime}$ over all triangles $t \in T_{G}$ and $t^{\prime} \in T_{H}$ is a complete edge-disjoint $K_{3} \times K_{3}$-packing in $G \times H$. The lemma follows as each $K_{3} \times K_{3}$ is $K_{3}$-decomposable.

Proof of Theorem 6.2. Consider the Shrikhande and $4 \times 4$ rook's graphs, $S$ and $R$, as in the proof of Theorem 6.1. Since $S \equiv_{2-\mathrm{WL}} R$, by Lemma 6.3 we also have $S^{k} \equiv_{2 \text {-WL }} R^{k}$, where the $k^{t h}$ power is with respect to the tensor product. To obtain the bound for $I D^{K_{3}}(n)$, it suffices, therefore, to prove that

$$
\rho\left(S^{k}\right)-\rho\left(R^{k}\right)=\Omega\left(v\left(R^{k}\right)^{1.25}\right)=\Omega\left(2^{5 k}\right) .
$$

Recall that $S$ is $K_{3}$-decomposable. By Lemma 6.4, $S^{k}$ is also decomposable and, hence, $\rho\left(S^{k}\right) \geq \rho\left(R^{k}\right)$. Let $\partial(G)$ denote the number of edges that remain not covered by an optimal triangle packing in $G$. Note that $\rho\left(S^{k}\right)-\rho\left(R^{k}\right)=\partial\left(R^{k}\right)$, and we will estimate the last value.

Obviously,

$$
\partial(G) \geq v_{\text {odd }}(G) / 2,
$$

where $v_{\text {odd }}(G)$ denotes the number of vertices of odd degree in $G$. Denote $K=\left(K_{4}\right)^{k}$. Since $v_{\text {odd }}(K)=v(K)=4^{k}$, we conclude that $\partial(K) \geq 2^{2 k-1}$. 
Note that $R=K_{4} \square K_{4}$, where $\square$ denotes the Cartesian product of graphs. It readily follows that $R$ is completely decomposable into 8 copies of $K_{4}$ and, therefore, $R^{k}$ is completely decomposable into $8^{k}$ copies of $K$. Every triangle $t$ in $R^{k}$ is included in one of these $K$-subgraphs. Indeed, let $t_{1}, \ldots, t_{k}$ be the projections of $t$ onto the $k$ coordinates. Each triangle $t_{i}$ is (uniquely) extendable to a $K_{4}=: K(i)$ in $R$. Therefore, $t$ belongs to $K(1) \times \cdots \times K(k)$.

It follows that

$$
\partial\left(R^{k}\right) \geq 8^{k} \partial(K) \geq 2^{3 k} 2^{2 k-1}=2^{5 k} / 2,
$$

yielding the desired bound (9).

\subsection{Domination number}

We conclude this section with a discussion of the domination number. Recall that, by Estimate (3) , the integrality gap of $\gamma_{f}$ over $n$-vertex graphs is bounded by $1+\ln n$, which is also an upper bound for the invariance ratio of $\gamma$. We now prove a lower bound that is tight up to a constant factor.

A circulant graph of $n$ vertices is a Cayley graph $\operatorname{Cay}\left(\mathbb{Z}_{n}, C\right)$ of the cyclic group $\mathbb{Z}_{n}$. Here, the connection set $C$ is a subset of $\mathbb{Z}_{n}$ such that $0 \notin C$ and $C=-C$. Two vertices $x, y \in \mathbb{Z}_{n}$ are adjacent in $\operatorname{Cay}\left(\mathbb{Z}_{n}, C\right)$ if $x-y \in C$. Let $q$ be a prime power such that $q \equiv 1(\bmod 4)$. The Paley graph on $q$ vertices is the circulant graph $\operatorname{Cay}\left(\mathbb{Z}_{q}, C_{q}\right)$ where $C_{q}$ is the multiplicative subgroup of $\mathbb{Z}_{q}^{*}$ formed by all quadratic residues modulo $q$.

Part 1 of Theorem 6.5 below implies that the integrality gap of the fractional domination number for Paley graphs is logarithmic. This was shown in [11] for random graphs. Problem 3.4 in [11] aks whether a logarithmic gap can be shown by an explicit construction. Our approach answers this in the affirmative as Paley graphs are explicitly constructed. It is actually not a big surprise that, using Paley graphs, one can replace a probabilistic argument in the situation like this. Indeed, it is well known that, in some precise sense, the Paley graphs have the same first-order properties as random graphs. Note in this respect that the property $\gamma(G)>k$ of a graph $G$ is clearly expressible in first-order logic. The proof also uses the 1-WLinvariance of the fractional domination number.

\section{Theorem 6.5.}

1. Let $n$ be a prime power such that $n \equiv 1(\bmod 4)$, and $G_{n}$ denote the Paley graph on $n$ vertices. Then $\gamma\left(G_{n}\right) \geq\left(\frac{1}{2}-o(1)\right) \log _{2} n$ while $\gamma_{f}\left(G_{n}\right) \leq 2$.

2. For infinitely many $n$, there are 1-WL-equivalent $n$-vertex graphs $G_{n}$ and $H_{n}$ such that $\gamma\left(G_{n}\right) / \gamma\left(H_{n}\right) \geq\left(\frac{1}{4}-o(1)\right) \log _{2} n$.

Proof. The $k$-extension property says that, for every two disjoint sets of vertices $X$ and $Y$ with $|X \cup Y| \leq k$ (where one of $X$ and $Y$ can be empty), there is a vertex $z \notin X \cup Y$ adjacent to each $x \in X$ and to no $y \in Y$. It is known [6, [8] that the 
Paley graph $G_{n}$ has the $k$-extension property for each $k$ such that $n>k^{2} 2^{2 k-1}$. The last condition is true, in particular, for $k=k(n)$ where

$$
k(n)=\left\lceil\frac{1}{2} \log _{2} n-\log _{2} \log _{2} n\right\rceil .
$$

Note that the $k$-extension property implies that the domination number exceeds $k$. It follows that

$$
\gamma\left(G_{n}\right)>k(n) \geq \frac{1}{2} \log _{2} n-\log _{2} \log _{2} n .
$$

Now, let $n=4 s+1$ and consider the circulant graph $H_{n}=\operatorname{Cay}\left(\mathbb{Z}_{n},\{ \pm 1, \ldots, \pm s\}\right)$. The set $\{0,2 s\}$ is dominating in $H_{n}$, which implies $\gamma\left(H_{n}\right)=2$. Since $G_{n}$ and $H_{n}$ are regular graphs of the same degree, $G_{n} \equiv_{1-\mathrm{WL}} H_{n}$. By Rubalcaba's result on the 1-WL-invariance of the fractional domination number (see Section 4.2), we therefore have

$$
\gamma_{f}\left(G_{n}\right)=\gamma_{f}\left(H_{n}\right) \leq \gamma\left(H_{n}\right)=2
$$

which implies Part 1.

The pair of graphs $G_{n}$ and $H_{n}$ yields also a proof of Part 2.

\section{Conclusion}

We have studied Weisfeiler-Leman invariance of the fractional packing number $\pi_{f}^{F}$ and its edge-disjoint variant $\rho_{f}^{F}$. As a starting point of our analysis, we have shown that the fractional matching number of a hypergraph is 1-WL-invariant, where a hypergraph (an instance of the Set Packing problem) is represented by its incidence graph. For a pattern graph $F$ with $\ell$ vertices, this already implies the $(2 \ell-1)$-WLinvariance of $\pi_{f}^{F}$ (see Corollary 4.3). Our main result, Theorem 5.3, is more precise. It shows for pattern graphs $F$ of hereditary treewidth $k$ that $\pi_{f}^{F}$ is $k$-WL-invariant. This is optimal in some cases, for example, when $F=K_{3}$ or $F=K_{1, s}$. The latter case, i.e., when $F$ is a star, includes the fractional matching number and adds to the list of fractional graph parameters invariant under fractional isomorphisms.

An important motivation for the study of WL-invariance is that this concept can be applied to showing lower bounds on the integrality gap of a fractional graph parameter $\pi_{f}$ :

- we first prove that $\pi_{f}$ is $k$-WL-invariant for an integer $k$;

- then, we estimate the $k$-WL-invariance ratio of $\pi$ from below, which provides us with a lower bound for the integrality gap of $\pi_{f}$.

Interestingly, this approach yields tight bounds in some cases like for the fractional matching number $\nu_{f}$, the fractional cover number $\tau_{f}$, the fractional domination

number $\gamma_{f}$, and the fractional edge-disjoint triangle packing number $\rho_{f}^{K_{3}}$. While the 
first two examples are based on simple graph sequences 4 , the last two cases are considered in Theorems 6.5 and 6.1 respectively. It is worth mentioning that, for all these parameters, the lower bounds for the invariance ratio are shown using explicit graphs or graph sequences, which in the case of the fractional domination number gives a solution of [11, Problem 3.4].5 In general, the connection between the integrality gap and invariance ratio is an intriguing question that merits further study. Are these two characteristics close to each other for all WL invariant graph parameters?

Another important question, whose discussion is initiated in Section 6.2, is whether the 2-WL-invariance of $\rho_{f}^{K_{3}}$ can be used for obtaining tight bounds on the additive integrality gap of this parameter.

\section{Acknowledgement.}

We thank Glenn Chappell for sending us his manuscript [10].

\section{References}

[1] M. Anderson, A. Dawar, and B. Holm. Solving linear programs without breaking abstractions. J. ACM, 62(6):48:1-48:26, 2015.

[2] A. Atserias and A. Dawar. Definable inapproximability: New challenges for duplicator. In 27th EACSL Annual Conference on Computer Science Logic (CSL'18), volume 119 of LIPIcs, pages 7:1-7:21. Schloss Dagstuhl - LeibnizZentrum für Informatik, 2018.

[3] A. Atserias and E. N. Maneva. Sherali-Adams relaxations and indistinguishability in counting logics. SIAM J. Comput., 42(1):112-137, 2013.

[4] L. Babai. Graph isomorphism in quasipolynomial time. In Proceedings of the 48th Annual ACM Symposium on Theory of Computing (STOC'16), pages 684697, 2016.

[5] L. Babai, P. Erdős, and S. M. Selkow. Random graph isomorphism. SIAM Journal on Computing, 9(3):628-635, 1980.

[6] A. Blass, G. Exoo, and F. Harary. Paley graphs satisfy all first-order adjacency axioms. Journal of Graph Theory, 5(4):435-439, 1981.

[7] A. Blass, Y. Gurevich, and S. Shelah. On polynomial time computation over unordered structures. J. Symb. Log., 67(3):1093-1125, 2002.

\footnotetext{
${ }^{4}$ For $\nu_{f}$, it is enough to consider $G=C_{6 s}$ and $H=2 s C_{3}$, as discussed in Section 11. For $\tau_{f}$, consider the pair of 1-WL-equivalent graphs $G=2 K_{s} \cup s K_{2}$ and $H=K_{s, s}$, where $G$ is the $2 s$-vertex graph obtained from two vertex-disjoint $s$-cliques by adding a perfect matching between them, and note that $\tau(G)=2 s-2$ while $\tau(H)=s$.

${ }^{5}$ We have just learned that a different solution is given in [10].
} 
[8] B. Bollobás and A. Thomason. Graphs which contain all small graphs. Eur. J. Comb., 2(1):13-15, 1981.

[9] J. Cai, M. Fürer, and N. Immerman. An optimal lower bound on the number of variables for graph identifications. Combinatorica, 12(4):389-410, 1992.

[10] G. Chappell. Constructing graphs with domination number and fractional domination number far apart. An unpublished manuscript, 2017.

[11] G. Chappell, J. Gimbel, and C. Hartman. Approximations of the domination number of a graph. J. Combin. Math. Combin. Comput., 104:287-297, 2018.

[12] M. Chlebík and J. Chlebíková. Approximation hardness of dominating set problems in bounded degree graphs. Inf. Comput., 206(11):1264-1275, 2008.

[13] A. Dawar. The nature and power of fixed-point logic with counting. SIGLOG News, 2(1):8-21, 2015.

[14] A. Dawar, S. Severini, and O. Zapata. Pebble games and cospectral graphs. Electronic Notes in Discrete Mathematics, 61:323-329, 2017.

[15] D. Dor and M. Tarsi. Graph decomposition is NP-complete: A complete proof of Holyer's conjecture. SIAM J. Comput., 26(4):1166-1187, 1997.

[16] F. Dross. Fractional triangle decompositions in graphs with large minimum degree. SIAM J. Discrete Math., 30(1):36-42, 2016.

[17] Z. Dvořák. On recognizing graphs by numbers of homomorphisms. Journal of Graph Theory, 64(4):330-342, 2010.

[18] H.-D. Ebbinghaus and J. Flum. Finite model theory. Springer Monographs in Mathematics. Springer-Verlag, Berlin, 2006.

[19] T. Feder and C. S. Subi. Packing edge-disjoint triangles in given graphs. Technical Report 13, Electronic Colloquium on Computational Complexity (ECCC), 2012.

[20] M. Fortin. $F O=F O^{3}$ for linear orders with monotone binary relations. In 46th International Colloquium on Automata, Languages, and Programming (ICALP'19), volume 132 of LIPIcs, pages 116:1-116:13. Schloss Dagstuhl Leibniz-Zentrum fuer Informatik, 2019.

[21] F. Fuhlbrück, J. Köbler, and O. Verbitsky. Local WL invariance and hidden shades of regularity. Technical report, arxiv.org/abs/2002.04590, 2020.

[22] Z. Füredi. Maximum degree and fractional matchings in uniform hypergraphs. Combinatorica, 1(2):155-162, 1981. 
[23] Z. Füredi. Matchings and covers in hypergraphs. Graphs and Combinatorics, 4(1):115-206, 1988.

[24] M. Fürer. On the power of combinatorial and spectral invariants. Linear Algebra and its Applications, 432(9):2373-2380, 2010.

[25] M. R. Garey and D. S. Johnson. Computers and Intractability: A Guide to the Theory of NP-Completeness. W. H. Freeman, 1979.

[26] M. Grohe, K. Kersting, M. Mladenov, and E. Selman. Dimension reduction via colour refinement. In Proc. of ESA'14, volume 8737 of Lecture Notes in Computer Science, pages 505-516. Springer, 2014. A full version in arxiv.org/abs/1307.5697.

[27] P. E. Haxell and V. Rödl. Integer and fractional packings in dense graphs. Combinatorica, 21(1):13-38, 2001.

[28] L. Hella. Logical hierarchies in PTIME. Inf. Comput., 129(1):1-19, 1996.

[29] C. A. J. Hurkens and A. Schrijver. On the size of systems of sets every $t$ of which have an SDR, with an application to the worst-case ratio of heuristics for packing problems. SIAM J. Discrete Math., 2(1):68-72, 1989.

[30] N. Immerman and D. Kozen. Definability with bounded number of bound variables. Inf. Comput., 83(2):121-139, 1989.

[31] R. Karp. Reducibility among combinatorial problems. In R. Miller, J. Thatcher, and J. Bohlinger, editors, Complexity of Computer Computations, The IBM Research Symposia Series, pages 85-103. Springer, Boston, MA, 1972.

[32] S. Kiefer and D. Neuen. The power of the Weisfeiler-Leman algorithm to decompose graphs. In 44th International Symposium on Mathematical Foundations of Computer Science (MFCS'19), volume 138 of LIPIcs, pages 45:1-45:15. Schloss Dagstuhl - Leibniz-Zentrum für Informatik, 2019.

[33] D. G. Kirkpatrick and P. Hell. On the complexity of general graph factor problems. SIAM J. Comput., 12(3):601-609, 1983.

[34] N. M. Kriege, F. D. Johansson, and C. Morris. A survey on graph kernels. Technical report, abs/1903.11835, 2019.

[35] L. Lovász. On the ratio of optimal integral and fractional covers. Discrete Mathematics, 13(4):383-390, 1975.

[36] L. Lovász. Large Networks and Graph Limits, volume 60 of Colloquium Publications. American Mathematical Society, 2012. 
[37] C. Morris, M. Ritzert, M. Fey, W. L. Hamilton, J. E. Lenssen, G. Rattan, and M. Grohe. Weisfeiler and Leman go neural: Higher-order graph neural networks. In 33rd AAAI Conference on Artificial Intelligence (AAAI'19), pages 4602-4609. AAAI Press, 2019.

[38] M. Otto. Bounded Variable Logics and Counting: A Study in Finite Models, volume 9 of Lecture Notes in Logic. Cambridge University Press, 2017.

[39] M. V. Ramana, E. R. Scheinerman, and D. Ullman. Fractional isomorphism of graphs. Discrete Mathematics, 132(1-3):247-265, 1994.

[40] R. R. Rubalcaba. Fractional Domination, Fractional Packings, and Fractional Isomorphisms of Graphs. PhD thesis, Auburn University, 2005.

[41] E. R. Scheinerman and D. H. Ullman. Fractional graph theory. A rational approach to the theory of graphs. Wiley: John Wiley \& Sons, 1997.

[42] G. Tinhofer. Graph isomorphism and theorems of Birkhoff type. Computing, $36: 285-300,1986$.

[43] B. Weisfeiler and A. Leman. The reduction of a graph to canonical form and the algebra which appears therein. NTI, Ser. 2, 9:12-16, 1968. English translation is available at https://www.iti.zcu.cz/wl2018/pdf/wl_paper_translation.pdf.

[44] R. Yuster. Integer and fractional packing of families of graphs. Random Struct. Algorithms, 26(1-2):110-118, 2005.

[45] R. Yuster. Combinatorial and computational aspects of graph packing and graph decomposition. Computer Science Review, 1(1):12-26, 2007. 